\title{
On the stationary points of the seismic reflection tomography and differential semblance functionals in laterally homogeneous media
}

\author{
Christiaan C. Stolk \\ Dept. of Computational \& Applied Mathematics, Rice University, 6100 Main St. - \\ MS 134, Houston, TX 77005-1892, USA \\ E-mail: cstolk@caam.rice.edu
}

\begin{abstract}
This paper concerns the determination of the reference medium (velocity model) in reflection seismology by optimization. Several objective functionals have been proposed, that attain their minimum or maximum at the correct medium. We study the local minima of two of these, the differential semblance and reflection tomography functionals, both depending on the traveltimes of reflected waves. It is assumed that the medium contains a single, horizontal reflector, and that it depends smoothly on the vertical coordinate above the reflector. We show that stationary points of both functionals are global minima when the medium above the reflector is non-constant. For reflection tomography we study the Hessian at a constant medium to show that all local minima are in fact global minima. To obtain these results we study the linearization of the map from medium properties to the traveltime of reflected waves around a non-constant medium.
\end{abstract}

AMS classification scheme numbers: $86 \mathrm{~A} 22,35 \mathrm{R} 30$

Submitted to: Inverse Problems 


\section{Introduction}

Most methods of imaging single reflection seismic data require the computation of a reference medium that gives an asymptotic approximation of the transmitted part of the waves. Migration methods use this reference medium to map the singularities in seismic data to the corresponding subsurface position, to produce an image of the discontinuities in the medium coefficients [1]. Computation of the reference medium relies essentially on the traveltime of reflected waves that is contained in the data. At present the accurate determination of the reference medium is major problem in seismic imaging.

Minimization of the differential semblance objective functional [2] is one method to find the reference medium. The differential semblance functional is one choice of objective functional related to migration velocity analysis [3], see [4] for a discussion and references on such methods. We will use the asymptotic expression for the differential semblance functional given in [5]. Because a global search for a minimum is generally to expensive, local optimization methods have to be used. In this paper we will therefore consider the question whether the differential semblance functional has local minima.

Our study of the dependence of the traveltime on the mediumcoefficients also yields results about seismic reflection tomography [6]. In this method the traveltimes of waves reflected from one or more major reflectors are estimated from the data, and then a medium is sought that minimizes the difference between modeled traveltimes observed traveltimes. The applicability of this method is limited by the fact that traveltimes have to be estimated from the data, contrary to differential semblance optimization.

We restrict ourselves to media that are laterally homogeneous, i.e. the medium coefficient depends only on the vertical coordinate. We assume our medium has a single reflector. This is a two dimensional problem. It can be applied to the case of several reflectors. Our main result is that for both the reflection tomography norm and the differential semblance norm the stationary points are global minima, provided the medium is nonconstant above the reflector (Corollary 4.1, Theorem 4.3). For the differential semblance norm we have to assume the medium is nonconstant on a somewhat smaller depth interval. In the case of a constant medium the stationary points of the reflection tomography functional cannot be a minimum unless it is global minimum, see Theorem 4.2. For reflection tomography it was noted in [7] that the least squares method need not converge to the solution when starting with a constant medium. The proof of our statements involves properties of the linearized inverse problem. In Theorem 3.2 we show that the range of the derivative of traveltime with respect to medium coefficient and reflector depth is dense in a set of even $C^{\infty}$ functions, in strong contrast with the linearization around a constant medium. The kernel is characterized in Theorem 3.1.

Our results builds on a number of results in the literature. In the paper [8] it was 
shown that for a horizontally layered medium in the limit $x_{1} \rightarrow 0$

$$
T\left(x_{1}\right)^{2}=T(0)^{2}+\frac{x_{1}^{2}}{c_{\mathrm{RMS}}^{2}},
$$

where $c_{\mathrm{RMS}}$ is the root mean square velocity (given by $c_{\mathrm{RMS}}^{2}=\frac{1}{d} \int_{0}^{\tilde{d}} \tilde{c}(s)^{2} \mathrm{~d} s$, where $\tilde{d}, \tilde{c}$ are as defined below). A uniqueness result for laterally homogeneous medium has been given in $[9,10,7]$ (see Section 2 below). It was found that it is precisely the distribution of the medium coefficient under Lebesgue measure that can be reconstructed uniquely from the traveltime function. For each interval of $c$ values the traveltime function determines the measure of the set of depth values such that $c$ is in the given interval, but it is impossible to determine their position. In $[10,7]$ also a least squares solution method is discussed.

For laterally varying media the linearized problem around a constant background with horizontal reflector has been considered [11, 12, 13, 14, 15, 16]. In [16] it has been shown that in that case the linearized forward operator has a large kernel that can be completely characterized. In this work we study the linearization around a nonconstant medium.

In [5] the question of the local minima of the differential semblance functional was considered, for laterally homogeneous medium and general reflectivity function (not a single reflector). However, in that paper the traveltimes are approximated using (1), which deviates from the correct (ray-theory) traveltimes. It was shown that in that approximation the stationary points of the differential semblance functional are global minima. Here we use the correct traveltimes to show this result for a single reflector.

The contents of the following sections is as follows. In section 2 expressions for the traveltime are given, and the uniqueness problem is discussed. Section 3 concerns the linearization of the map from medium coefficient and reflector depth to the traveltime function. In section 4 the results on the stationary points of the reflection tomography and differential semblance functionals are given.

\section{Notation}

Some notation will be used frequently in this paper. By $T: x_{1} \mapsto T\left(x_{1}\right)$ we denote the traveltime of a ray originating in the origin, reflected at depth $d$ and arriving at $\left(x_{1}, 0\right)$. Instead of $x_{1}$ the ray parameter, or the horizontal component of the slowness vector, can also be used to parametrize the traveltime function. There is a diffeomorphic map $X_{1}: p_{1} \mapsto X_{1}\left(p_{1}\right)$. We define $\tau$ by $\tau(p)=T\left(X_{1}\left(p_{1}\right)\right)$. The medium coefficient (soundspeed) will be denoted by $c: x_{2} \mapsto c\left(x_{2}\right)$. Because the square of the medium coefficient occurs often we define the function $\gamma$ by $\gamma\left(x_{2}\right)=c\left(x_{2}\right)^{2}$. We will assume that $c$ has upper and lower bounds, and in conjunction there is a bound $\alpha$ for the $p_{1}$ variable.

$$
C_{1} \leq c \leq C_{2}<\alpha^{-1} .
$$


The ray parameter will be bounded $-\alpha \leq p_{1} \leq \alpha$. There will also be bounds for the $x_{1}$ variable, that must depend on the bounds for $c$ and on the depth of the reflector or the time. We assume $-\hat{\alpha} \leq x_{1} \leq \hat{\alpha}$. In this symmetric situation the traveltime is an even function of $x_{1}$, or $p_{1}$.

It will be convenient in later sections to use a modified depth coordinate given by the traveltime along a vertical ray

$$
S\left(x_{2}\right)=\int_{0}^{x_{2}} c\left(x_{2}^{\prime}\right)^{-1} \mathrm{~d} x_{2}^{\prime} .
$$

We denote the reflector depth in the new coordinate by $\tilde{d}=S(d)$ and the medium by $\tilde{c}$ or $\tilde{\gamma}$, define by pull back, $\tilde{c}\left(S\left(x_{2}\right)\right)=c\left(x_{2}\right), \tilde{\gamma}\left(S\left(x_{2}\right)\right)=\gamma\left(x_{2}\right)$.

\section{Traveltime and the uniqueness problem}

The propagation of rays in time is described by the Hamilton system with Hamiltonian $H(x, p)=\frac{1}{2} c(x)^{2} p^{2}$. The Hamilton system is

$$
\frac{\partial x}{\partial t}=\frac{\partial H}{\partial p}=c(x)^{2} p, \quad \frac{\partial p}{\partial t}=-\frac{\partial H}{\partial x}=-\frac{1}{c(x)} \frac{\partial c}{\partial x} .
$$

Here $p=\left(p_{1}, p_{2}\right) \in \mathbb{R}^{2}$ is the slowness vector, $p_{1}$ is sometimes called the ray parameter. The slowness vector must satisfy

$$
c(x)\|p\|=1 .
$$

The projection of the solution on the $x$ coordinates is the ray.

In our case the medium coefficient depends only on $x_{2}$ and therefore $p_{1}$ is conserved. Together with (5) this implies that $p$ and hence $\frac{\partial x}{\partial t}$ is known for each $x$. This yields the following formulas for the traveltime and the horizontal position of a ray reflected of a horizontal reflector at depth $d$ as a function of the ray parameter (see [17], section 12.1).

$$
\begin{aligned}
\tau\left(p_{1}\right) & =2 \int_{0}^{d} \mathrm{~d} x_{2} c\left(x_{2}\right)^{-1}\left(1-c\left(x_{2}\right)^{2} p_{1}^{2}\right)^{-\frac{1}{2}}, \\
X_{1}\left(p_{1}\right) & =2 \int_{0}^{d} \mathrm{~d} x_{2} p_{1} c\left(x_{2}\right)\left(1-c\left(x_{2}\right)^{2} p_{1}^{2}\right)^{-\frac{1}{2}} .
\end{aligned}
$$

The factors 2 are because the reflected ray first travels downward, and then upward. These expressions are valid when $\left|p_{1}\right|<c\left(x_{2}\right)$ for all $x_{2} \in[0, d]$. Note that the traveltime is an analytic function. The map $p_{1} \mapsto X_{1}\left(p_{1}\right)$ is an analytic diffeomorphism. It follows that the traveltime function $x_{1} \mapsto T\left(x_{1}\right)$ is analytic as well.

From now on we use the notation $\gamma\left(x_{2}\right)=c\left(x_{2}\right)^{2}$. The traveltime function depends only on the medium through its distribution under Lebesgue measure. This is a measure (a positive distribution of order 0 ), that we will denote as $\gamma_{*} I_{[0, d]}$, i.e. the push forward of the indicator function $I_{[0, d]}$. It is defined by

$$
\left\langle\gamma_{*} I_{[0, d]}, \phi\right\rangle=\int \phi\left(\gamma\left(x_{2}\right)\right) I_{[0, d]} \mathrm{d} x_{2} .
$$


The traveltime is given by

$$
\tau\left(p_{1}\right)=2 \int \gamma_{*} I_{[0, d]}(y) y^{-\frac{1}{2}}\left(1-y p_{1}^{2}\right)^{-\frac{1}{2}} \mathrm{~d} y .
$$

It has been noted in the literature that the traveltime as a function of $p_{1}$ uniquely determines the distribution of $\gamma$. The same is true for the function $x_{1} \mapsto T\left(x_{1}\right)$ since $p_{1}=\frac{\partial T}{\partial x_{1}}$. This is mentioned in [9] where for the proof the reader is referred to a paper in Russian. An independent proof in English is given in [10, 7]. Given the preparations that we have done the uniqueness argument is not difficult. The moments of $y \mapsto\left(\gamma_{*} I_{[0, d]}\right)(y) y^{-\frac{1}{2}}$ are given by the derivatives of the traveltime function with respect to $p_{1}^{2}$ at $p_{1}^{2}=0$

$$
\frac{\partial^{k} \tau}{\partial\left(p_{1}^{2}\right)^{k}}(0)=2 \frac{1 \cdot 3 \cdot \ldots \cdot(2 k-1)}{2^{k}} \int \gamma_{*} I_{[0, D]}(y) y^{-\frac{1}{2}+k}
$$

Since $\gamma_{*} I_{[0, d]}(y) y^{-\frac{1}{2}}$ is compactly supported the moments determine this distribution. An argument for this is that when $I$ is a compact interval in $\mathbb{R}$, then the polynomials are dense in $C^{\infty}(I)$, and hence are a sufficiently large class of testfunctions to determine a distribution.

\section{Linearization}

For the computation of an approximate solution to the inverse problem it is important to know the properties of its linearization. We therefore study the derivative of the traveltime functions $T: x_{1} \mapsto T\left(x_{1}\right)$ and $\tau: p_{1} \mapsto \tau\left(p_{1}\right)$ with respect to the reflector depth $d$ and the medium parameter $\gamma$. The medium parameter $\gamma$ is assumed to be in $C^{\infty}([0, d])$. It is known that when $\gamma$ is constant then the range of the derivative is of dimension 2 and the kernel is large. We show that when $\gamma$ is nonconstant the situation is very different. The kernel is equal to $\{0\}$ when the map $x_{2} \mapsto \gamma\left(x_{2}\right)$ is injective (strictly monotonous), otherwise certain cancellations lead to a nonzero kernel. The range is dense in a space of even $C^{\infty}$ functions. In the next section this last result is used to obtain a result about the computation of solutions of the inverse problem through the minimization of certain objective functions.

The directional derivative of $T$ with respect to the mediumcoefficient $\gamma$ in the direction $\delta \gamma$ is will be denoted by $\frac{\delta T}{\delta \gamma} \delta \gamma$ and is defined as

$$
\frac{\delta T}{\delta \gamma} \delta \gamma=\left.\frac{\partial}{\partial \alpha} T(d, \gamma+\alpha \delta \gamma)\right|_{\alpha=0}
$$

Here in the right hand side we indicated the dependence of $T$ on the mediumcoefficient explicitly. We will usually omit the dependence on $d$ and the mediumcoefficient $\gamma$. By $\frac{\delta T}{\delta(d, \gamma)}$ we denote the linear map

$$
(\delta d, \delta \gamma) \mapsto \frac{\partial T}{\partial d} \delta d+\frac{\delta T}{\delta \gamma} \delta \gamma
$$


For the computations to follow we use the modified depth coordinate given in (3). The reflector depth in the new coordinate is denoted by $\tilde{d}=S(d)$ and the medium by $\tilde{c}$ or $\tilde{\gamma}$. The derivative of the map $(d, \gamma) \mapsto(\tilde{d}, \tilde{\gamma})$ is given by

$$
\begin{aligned}
& \frac{\partial \tilde{d}}{\partial d} \delta d+\frac{\delta \tilde{d}}{\delta \gamma} \delta \gamma=c(d)^{-1} \delta d-\int_{0}^{d} \gamma\left(x_{2}\right)^{-\frac{3}{2}} \delta \gamma\left(x_{2}\right) \mathrm{d} x_{2} \\
& \frac{\delta \tilde{\gamma}}{\delta \gamma} \delta \gamma\left(S\left(x_{2}\right)\right)=\delta \gamma\left(x_{2}\right)-\frac{\partial \gamma}{\partial x_{2}}\left(x_{2}\right) \gamma\left(x_{2}\right)^{\frac{1}{2}} \int_{0}^{x_{2}}\left(-\frac{1}{2}\right) \gamma\left(x_{2}^{\prime}\right)^{-\frac{3}{2}} \delta \gamma\left(x_{2}^{\prime}\right) \mathrm{d} x_{2}^{\prime} .
\end{aligned}
$$

The derivative of the inverse map exists as well, which implies that the calculations may be done using the modified coordinates.

In the new coordinates the formula for the traveltime is slightly simplified

$$
\tau\left(p_{1}\right)=2 \int_{0}^{\tilde{d}}\left(1-\tilde{\gamma}(s) p_{1}^{2}\right)^{-\frac{1}{2}} \mathrm{~d} s=\int \tilde{\gamma}_{*} I_{[0, \tilde{d}]}(y)\left(1-y p_{1}^{2}\right)^{-\frac{1}{2}} \mathrm{~d} y .
$$

This leads to the following expressions for $\frac{\partial \tau}{\partial \tilde{d}}$ and $\frac{\delta \tau}{\delta \tilde{\gamma}}$

$$
\begin{aligned}
\frac{\partial \tau}{\partial \tilde{d}}\left(p_{1}\right) & =2\left(1-p_{1}^{2} \tilde{\gamma}(\tilde{d})\right)^{-\frac{1}{2}}, \\
\frac{\delta \tau}{\delta \tilde{\gamma}} \delta \tilde{\gamma}\left(p_{1}\right) & =\int_{0}^{\tilde{d}} p_{1}^{2}\left(1-p_{1}^{2} \tilde{\gamma}(s)\right)^{-\frac{3}{2}} \delta \tilde{\gamma}(s) \mathrm{d} s .
\end{aligned}
$$

Using that $T\left(X_{1}\left(p_{1}\right)\right)=\tau\left(p_{1}\right)$, that both $\tau$ and $X_{1}$ depend on $\tilde{d}$ and $\tilde{\gamma}$, and that $\frac{\partial T}{\partial x_{1}}\left(X_{1}\left(p_{1}\right)\right)=p_{1}$, we find that

$$
\begin{aligned}
\frac{\delta T}{\delta(\tilde{d}, \tilde{\gamma})}\left(X_{1}\left(p_{1}\right)\right) & =\frac{\delta \tau}{\delta(\tilde{d}, \tilde{\gamma})}\left(p_{1}\right)-\frac{\partial T}{\partial x_{1}}\left(X_{1}\left(p_{1}\right)\right) \frac{\delta X_{1}}{\delta(\tilde{d}, \tilde{\gamma})}\left(p_{1}\right) \\
& =\frac{\delta}{\delta(\tilde{d}, \tilde{\gamma})}\left(2 \int_{0}^{\tilde{d}}\left(1-\tilde{\gamma}(s) p_{1}^{2}\right)^{\frac{1}{2}}\right) \mathrm{d} s
\end{aligned}
$$

When $\tilde{\gamma}$ is constant $\frac{\delta \tau}{\delta(\tilde{d}, \tilde{\gamma})}$ is proportional to

$$
a\left(1-\gamma p_{1}^{2}\right)^{-\frac{1}{2}}+b p_{1}^{2}\left(1-\gamma p_{1}^{2}\right)^{-\frac{3}{2}}
$$

where $\gamma$ denotes the constant value of the function $\gamma$. Therefore range $\frac{\delta T}{\delta(\tilde{d}, \tilde{\gamma})}$ is of dimension 2. The kernel is given by

$$
\left\{(0, \delta \tilde{\gamma}) \mid \int_{0}^{\tilde{d}} \delta \tilde{\gamma}(s) \mathrm{d} s=0\right\}
$$

This is well known, see for instance $[7,16]$.

We show that for media with smoothly varying, nonconstant coefficient the situation is very different. We first study the kernel of $\frac{\delta T}{\delta(\tilde{d}, \tilde{\gamma})}$, after that we study the range.

Theorem 3.1 Assume $\tilde{\gamma} \in C^{\infty}([0, \tilde{d}])$ satisfies (2). Then

$$
\operatorname{ker} \frac{\delta T}{\delta(\tilde{d}, \tilde{\gamma})}=\operatorname{ker} \frac{\delta \tau}{\delta(\tilde{d}, \tilde{\gamma})}=\left\{(0, f) \mid \tilde{\gamma}_{*} f=0\right\}
$$

where $\tilde{\gamma}_{*}$ is the push forward $C^{\infty}([0, \tilde{d}]) \rightarrow{D^{\prime}}^{0}(\mathbb{R})$. In particular the maps $\frac{\delta T}{\delta(\tilde{d}, \tilde{\gamma})}, \frac{\delta \tau}{\delta(\tilde{d}, \tilde{\gamma})}$ are injective if and only if the map $s \mapsto \gamma^{*}(s)$ is injective (strictly monotonous). 
Proof We first prove the result for $\tau$. At $p_{1}=0$ the following is true

$$
\frac{\partial \tau}{\partial \tilde{d}}\left(p_{1}=0\right)=2, \quad \frac{\delta \tau}{\delta \tilde{\gamma}}\left(p_{1}=0\right)=0
$$

hence $\delta \tilde{d}=0$ in the kernel. It follows from (12) and the uniqueness argument in the previous section that the map $\tilde{\gamma} \mapsto \tau$ is the composition of the map $\tilde{\gamma} \mapsto \tilde{\gamma}_{*} I_{[0, \tilde{d}]}$ and a linear, injective map $\tilde{\gamma}_{*} I_{[0, \tilde{d}]} \mapsto \tau$. Therefore $(0, \delta \tilde{\gamma}) \in \operatorname{ker} \frac{\delta \tau}{\delta(\tilde{d}, \tilde{\gamma})}$ if and only if $\delta \tilde{\gamma}$ is in the kernel of the linearization of the push forward, that is

$$
\left.\frac{\partial}{\partial \alpha}(\tilde{\gamma}+\alpha \delta \tilde{\gamma})_{*} \lambda\right|_{\alpha=0}=0
$$

When acting on a testfunction $\phi \in C^{\infty}(\mathbb{R})$ this equals

$$
\left.\frac{\partial}{\partial \alpha} \int_{0}^{\tilde{d}} \phi(\tilde{\gamma}(s)+\alpha \delta \tilde{\gamma}(s)) \mathrm{d} s\right|_{\alpha=0}=\int_{0}^{\tilde{d}} \delta \tilde{\gamma}(s) \frac{\partial \phi}{\partial y}(\tilde{\gamma}(s)) \mathrm{d} s=\left\langle\tilde{\gamma} * \delta \tilde{\gamma}, \frac{\partial \phi}{\partial y}\right\rangle
$$

Since $\tilde{\gamma}_{*} \delta \tilde{\gamma}$ has compact support this proves the first part of the theorem for $\tau$. The expression (15) for $\frac{\delta T}{\delta(\tilde{d}, \tilde{\gamma})}$ is similar to the expression for $\frac{\delta \tau}{\delta(\tilde{d}, \tilde{\gamma})}$. The same arguments can be applied, and since the map $p_{1} \mapsto X_{1}\left(p_{1}\right)$ is a diffeomorphism the result for $T$ follows.

The condition $\tilde{\gamma}_{*} f=0$ may also be stated as

$$
\int_{\tilde{\gamma}^{-1}(E)} \delta \tilde{\gamma}(s) \mathrm{d} s=0
$$

for all measurable subsets $E$ of $\mathbb{R}$. Recall that $\tilde{\gamma}$ is smooth. When $\tilde{\gamma}$ is injective then this implies $\delta \tilde{\gamma}=0$. Otherwise there exist two disjoint intervals $I_{i}=\left[a_{i}, b_{i}\right], i=1,2$ in $[0, \tilde{d}]$ and a diffeomorphism $\Phi: I_{1} \rightarrow I_{2}$ such that $\tilde{\gamma}(s)=\tilde{\gamma}(\Phi(s))$ for $s \in I_{1}$. This can be used to construct nonzero $\delta \tilde{\gamma}$ such that (16) is satisfied.

The range of the derivative of $T$ with respect to $\tilde{d}, \tilde{\gamma}$ is given by

$$
\text { range }\left(\frac{\delta T}{\delta(\tilde{d}, \tilde{\gamma})}\right)=\left\{\frac{\partial T}{\partial \tilde{d}} \delta \tilde{d}+\frac{\delta T}{\delta \tilde{\gamma}} \delta \tilde{\gamma} \mid \delta \tilde{d} \in \mathbb{R}, \delta \tilde{\gamma} \in C^{\infty}([0, d])\right\}
$$

We have the following result. By $C_{\text {even }}^{\infty}([-\beta, \beta])$ we denote the set of smooth even functions on $[-\beta, \beta]$.

Theorem 3.2 Let $\tilde{\gamma} \in C^{\infty}([0, \tilde{d}])$ be bounded as in (2), and nonconstant. Then the range of $\frac{\delta T}{\delta(d, \tilde{\gamma})}$ and the range of $\frac{\delta \tau}{\delta(d, \tilde{\gamma})}$ are dense in $C_{\mathrm{even}}^{\infty}([-\hat{\alpha}, \hat{\alpha}]), C_{\mathrm{even}}^{\infty}([-\alpha, \alpha])$.

The proof requires a few intermediate results on the $(\tilde{d}, \tilde{\gamma})$ derivatives of expressions of the form

$$
G=\int_{0}^{\tilde{d}} g\left(\tilde{\gamma}(s), p_{1}\right) \mathrm{d} s
$$


These are given by

$$
\begin{aligned}
\frac{\delta G}{\delta \tilde{\gamma}} \delta \tilde{\gamma} & =\int_{0}^{\tilde{d}} \frac{\partial g}{\partial y}\left(\tilde{\gamma}(s), p_{1}\right) \delta \tilde{\gamma}(s) \mathrm{d} s \\
\frac{\partial G}{\partial \tilde{d}} & =g\left(\tilde{\gamma}(\tilde{d}), p_{1}\right) .
\end{aligned}
$$

Lemma 3.3 Let $f \in C^{\infty}([0, \tilde{d}])$. There are $\delta \tilde{d} \in \mathbb{R}$ and $\delta \tilde{\gamma} \in C^{\infty}([0, \tilde{d}])$ such that

$$
\frac{\partial G}{\partial \tilde{d}} \delta \tilde{d}+\frac{\delta G}{\delta \tilde{\gamma}} \delta \tilde{\gamma}=\int_{0}^{\tilde{d}} g\left(\tilde{\gamma}(s), p_{1}\right) f(s) \mathrm{d} s .
$$

Proof Let

$$
\delta \tilde{\gamma}(s)=-\frac{\partial \tilde{\gamma}}{\partial s}(s) \int_{0}^{s} f\left(s^{\prime}\right) \mathrm{d} s^{\prime}, \quad \delta \tilde{d}=\int_{0}^{\tilde{d}} f(s) \mathrm{d} s .
$$

Entering this in equations (18) and (17) we find that

$$
\frac{\partial G}{\partial \tilde{d}} \delta \tilde{d}+\frac{\delta G}{\delta \tilde{\gamma}} \delta \tilde{\gamma}=g\left(\tilde{\gamma}(\tilde{d}), p_{1}\right)\left(\int_{0}^{\tilde{d}} f(s)\right)-\int_{0}^{\tilde{d}} \frac{\partial \tilde{\gamma}}{\partial s} \frac{\partial g}{\partial y}\left(\tilde{\gamma}(s), p_{1}\right)\left(\int_{0}^{s} f\left(s^{\prime}\right) \mathrm{d} s^{\prime}\right) \mathrm{d} s .
$$

By partial integration this is equal to (19).

Lemma 3.4 Let $y_{\min }=\min _{s \in[0, \tilde{d}]} \tilde{\gamma}(s), y_{\max }=\max _{s \in[0, \tilde{d}]} \tilde{\gamma}(s)$. Let $h \in C_{0}^{\infty}\left(\left[y_{\min }, y_{\max }\right]\right)$. There is $f \in C_{0}^{\infty}([0, \tilde{d}])$ such that

$$
\int_{0}^{\tilde{d}} g\left(\tilde{\gamma}(s), p_{1}\right) f(s) \mathrm{d} s=\int_{y_{\min }}^{y_{\max }} g\left(y, p_{1}\right) h(y) \mathrm{d} y
$$

Proof Suppose that $\gamma$ takes its minimum and its maximum at $s_{\min }, s_{\max }$ respectively. Let

$$
f(s)= \begin{cases}0 & s \text { outside }\left[s_{\min }, s_{\max }\right] \\ \pm \frac{\partial \tilde{\gamma}}{\partial s} h(\tilde{\gamma}(s)) & s \in\left[s_{\min }, s_{\max }\right]\end{cases}
$$

where the $+\operatorname{sign}$ is in case $s_{\min }<s_{\max }$ and the $-\operatorname{sign}$ in case $s_{\min }>s_{\max }$, and $\left[s_{\min }, s_{\max }\right]$ is the interval with as endpoints $s_{\min }, s_{\max }$.

Let $\phi \in C_{0}^{\infty}(\mathbb{R})$ be supported in $[-1,1]$, nonnegative, even, with integral equal to 1. Define by $\phi_{\epsilon}$ by $\phi_{\epsilon}(y)=\epsilon^{-1} \phi\left(\epsilon^{-1} y\right)$.

Lemma 3.5 Let $g:\left(y, p_{1}\right) \mapsto g\left(y, p_{1}\right) \in C^{\infty}\left(\left[y_{\min }, y_{\max }\right] \times[-\alpha, \alpha]\right)$. Let $y_{\min }<y_{0}<$ $y_{\text {max }}$.

$$
\lim _{\epsilon \rightarrow 0} \frac{\partial^{j}}{\partial p_{1}^{j}}\left(\int_{y_{\min }}^{y_{\max }} g\left(y, p_{1}\right)(-1)^{k} \frac{\partial^{k} \phi_{\epsilon}}{\partial y^{k}}\left(y-y_{0}\right) \mathrm{d} y-\frac{\partial^{k} g}{\partial y^{k}}\left(y_{0}, p_{1}\right)\right)=0
$$

uniformly for $p_{1} \in[-\alpha, \alpha]$ and $j$ in any finite subset of $\mathbb{N}$. 
Proof Assume $\epsilon$ sufficiently small, so that $y_{\min }<y_{0}-\epsilon, y_{0}+\epsilon<y_{\max }$. By integration by parts

$$
\begin{aligned}
\int_{y_{\min }}^{y_{\max }} & g\left(y, p_{1}\right)(-1)^{k} \frac{\partial^{k} \phi_{\epsilon}}{\partial y^{k}}\left(y-y_{0}\right)=\int \frac{\partial^{k} g}{\partial y^{k}}\left(y, p_{1}\right) \phi\left(y-y_{0}\right) \mathrm{d} y \\
= & \frac{\partial^{k} g}{\partial y^{k}}\left(y_{0}, p_{1}\right)+\int\left(\frac{\partial^{k} g}{\partial y^{k}}\left(y, p_{1}\right)-\frac{\partial^{k} g}{\partial y^{k}}\left(y_{0}, p_{1}\right)\right) \phi_{\epsilon}\left(y-y_{0}\right) \mathrm{d} y .
\end{aligned}
$$

Since $\frac{\partial^{k} g}{\partial y^{k}}\left(y, p_{1}\right)$ and its $p_{1}$ derivatives are continuous uniformly in $p_{1}$ the second term in the right hand side converges to 0 uniformly when $\epsilon \rightarrow 0$.

Proof of Theorem 3.2 We first prove this for $\tau$. Let $g\left(y, p_{1}\right)=2\left(1-y p_{1}^{2}\right)^{-\frac{1}{2}}$. The derivatives of $g$ with respect to $y$ are given by

$$
\frac{\partial^{k} g}{\partial y^{k}}\left(y, p_{1}\right)=2 \frac{1 \cdot 3 \cdot \ldots \cdot(2 k-1)}{2^{k}}\left(1-y p_{1}^{2}\right)^{-\frac{1}{2}}\left(p\left(1-y p_{1}^{2}\right)^{-\frac{1}{2}}\right)^{2 k}
$$

By the lemmas above there are $\delta \tilde{d}, \delta \tilde{\gamma}$ such that these derivatives evaluated at some $y_{0}$ are approximated in $C^{l}$ norm, any $l$. It is therefore sufficient to show that the set of functions spanned by $\left\{p_{1} \mapsto\left(1-y_{0} p_{1}^{2}\right)^{-\frac{1}{2}}\left(p\left(1-y_{0} p_{1}^{2}\right)^{-\frac{1}{2}}\right)^{2 k}\right\}_{k \geq 0}$ is dense in $C_{\text {even }}^{\infty}([-\alpha, \alpha])$.

Let $\beta>0$. The sequence of polynomials $\left\{q^{k}\right\}_{k \geq 0}$ spans a dense set in $C^{\infty}([-\beta, \beta])$. Each $f \in C^{\infty}([-\beta, \beta])$ is the sum of its even and odd parts $f_{\text {even }}(q)=\frac{1}{2}\left(f_{k}(q)+f_{k}(-q)\right)$ and $f_{k, \text { odd }}(q)=\frac{1}{2}\left(f_{k}(q)-f_{k}(-q)\right)$. When $f_{k}$ is a sequence of polynomials converging to $f$, then its even (odd) part, consisting of even (odd) order terms, converge to the even (odd) part of $f$. It follows that the polynomials consisting of even order terms span a dense set in $C_{\text {even }}^{\infty}([-\beta, \beta])$ and the polynomials consisting of odd order terms span a dense set in $C_{\text {odd }}^{\infty}([-\beta, \beta])$.

Define a diffeomorphism $p \mapsto q(p)$ from $[-\alpha, \alpha]$ to some interval $[-\beta, \beta]$ by

$$
q(p)=p\left(1-y_{0} p^{2}\right)^{-\frac{1}{2}}
$$

The pull back $q^{*}$ is a homeomorphism from the space $C_{\text {even }}^{\infty}([-\beta, \beta])$ to $C_{\text {even }}^{\infty}([-\alpha, \alpha])$, i.e. it is continuous and has continuous inverse. Let $M$ be the multiplication operator

$$
f(p) \mapsto\left(1-y_{0} p^{2}\right)^{-\frac{1}{2}} f(p)
$$

The operator $M$ is a homeomorphism $C_{\text {even }}^{\infty}([-\alpha, \alpha]) \rightarrow C_{\text {even }}^{\infty}([-\alpha, \alpha])$. The set spanned by $\left\{p_{1} \mapsto \frac{\partial^{k} g}{\partial y^{k}}\left(y_{0}, p_{1}\right)\right\}_{k \geq 0}$ is the image of the homeomorphism $M \circ q^{*}$ of the even polynomials in $C^{\infty}([-\beta, \beta])$, and is therefore dense in $C_{\text {even }}^{\infty}([-\alpha, \alpha])$.

Using (15) we can now do the same analysis for $T$ as for $\tau$, except that $g\left(y, p_{1}\right)=$ $2\left(1-y p_{1}^{2}\right)^{-\frac{1}{2}}$ has to be replaced with $h\left(y, p_{1}\right)=2\left(1-y p_{1}^{2}\right)^{\frac{1}{2}}$. Using in addition that $p_{1} \mapsto X_{1}\left(p_{1}\right)$ is a diffeomorphism we find the claim for $\frac{\delta T}{\delta(\tilde{d}, \tilde{\gamma})}$. 


\section{Solving the inverse problem through optimization}

The reconstruction problem can be formulated as an optimization problem. Suppose traveltime data $x_{1} \mapsto T\left(x_{1} ; d^{*}, \gamma^{*}\right)$ are given from an a priori unknown medium $\gamma^{*}$, with reflector at depth $d^{*}$. A reconstruction $\gamma, d$ should minimize the functional

$$
K(d, \gamma)=\int_{-\hat{\alpha}}^{\hat{\alpha}}\left(T\left(x_{1} ; d, \gamma\right)-T\left(x_{1} ; d^{*}, \gamma^{*}\right)^{2} \mathrm{~d} x_{1} .\right.
$$

Another objective function is the differential semblance objective function given below. This raises questions about the properties of the objective function, in particular whether it has local minima. When $\gamma$ is constant it has been observed that stationary points that are not global minima may appear in (20), see [7] (this follows from the fact that $\operatorname{ker} \frac{\delta T}{\delta(d, \gamma)}$ is two dimensional). It will follow from the results of the previous section that, when $\gamma$ is $C^{\infty}$ and nonconstant, any stationary point is a global minimum, and hence yields a (possibly nonunique) solution to the inverse problem. We will show that the same is true for the differential semblance norm. When $\gamma$ is constant, then a stationary point of $K$ is either a global minimum, or the Hessian of $K$ has a negative eigenvalue, so that it is not a minimum, but a saddle point or a local maximum.

The derivative of $K$ with respect to $(d, \gamma)$ is essentially given by the inner product of $\left(T\left(x_{1} ; d, \gamma\right)-T\left(x_{1}, d^{*} \gamma^{*}\right)\right)$ and $\frac{\delta T}{\delta \gamma} \delta \gamma\left(x_{1} ; d, \gamma\right)+\frac{\partial T}{\partial d} \delta d\left(x_{1} ; d, \gamma\right)$

$$
\begin{aligned}
\frac{\partial K}{\partial d} \delta d+\frac{\delta K}{\delta \gamma} \delta \gamma= & 2 \int_{-\hat{\alpha}}^{\hat{\alpha}}\left(T\left(x_{1} ; d, \gamma\right)-T\left(x_{1} ; d^{*}, \gamma^{*}\right)\right) \\
& \times\left(\frac{\partial T}{\partial d} \delta d\left(x_{1} ; d, \gamma\right)+\frac{\delta T}{\delta \gamma} \delta \gamma\left(x_{1} ; d, \gamma\right)\right) \mathrm{d} x_{1}
\end{aligned}
$$

Theorem 3.2 therefore has the following corollary. We define the $p_{1}$ equivalent of (20) by

$$
\hat{K}(d, \gamma)=\int_{-\alpha}^{\alpha}\left(\tau\left(p_{1} ; d, \gamma\right)-\tau\left(p_{1} ; d^{*}, \gamma^{*}\right)^{2} \mathrm{~d} p_{1} .\right.
$$

Corollary 4.1 Let $\gamma \in C^{\infty}([0, d])$ be bounded as in (2), and nonconstant. Then

$$
\frac{\delta K}{\delta(d, \gamma)}(d, \gamma)=0
$$

implies that $(d, \gamma)$ is a solution to the inverse problem, i.e. $T\left(x_{1} ; d, \gamma\right)=$ $T\left(x_{1} ; d^{*}, \gamma^{*}\right), x_{1} \in[-\hat{\alpha}, \hat{\alpha}]$. The same is true for $\hat{K}$ when $x_{1}$ is replaced by $p_{1} \in[-\alpha, \alpha]$ and $T$ by $\tau$.

We show that in the case that $\gamma$ is constant there are no minima that are not global minima. In the following we will write $T$ for the function $x_{1} \mapsto T\left(x_{1} ; d, \gamma\right)$ and $T^{*}$ for the function $x_{1} \mapsto T\left(x_{1} ; d^{*}, \gamma^{*}\right)$, and similar for $\tau, \tau^{*}$.

Theorem 4.2 Suppose $\tilde{\gamma}$ is constant, $\frac{\delta K}{\delta(d, \gamma)}=0$ and $T \neq T^{*}$, then the Hessian $\frac{\delta^{2} K}{\delta(d, \gamma)^{2}}$ has a negative eigenvalue. The same is true for $\hat{K}$, with $T, T^{*}$ replaced by $\tau, \tau^{*}$. 
Proof We use the modified depth coordinate $s$ and the modified medium parameters $\tilde{d}, \tilde{\gamma}$. Using that $(\gamma, d)$ is a stationary point of $K$ and that $\frac{\delta(d, \gamma)}{\delta(\tilde{d}, \tilde{\gamma})}$ is invertible (see (11)) one can show that this can be done.

We prove the statement first for the $p_{1}$ case. The second derivative of $\hat{K}$ with respect to $\tilde{\gamma}$ is given by

$$
\frac{\delta^{2} \hat{K}}{\delta \tilde{\gamma}^{2}} \delta \tilde{\gamma} \delta \tilde{\gamma}^{\prime}=2 \int_{-\alpha}^{\alpha} \frac{\delta^{2} \tau}{\delta \tilde{\gamma}^{2}} \delta \tilde{\gamma} \delta \tilde{\gamma}^{\prime}\left(\tau\left(p_{1}\right)-\tau^{*}\left(p_{1}\right)\right) \mathrm{d} p_{1}+2 \int_{-\alpha}^{\alpha} \frac{\delta \tau}{\delta \tilde{\gamma}} \delta \tilde{\gamma}\left(p_{1}\right) \frac{\delta \tau}{\delta \tilde{\gamma}} \delta \tilde{\gamma}^{\prime}\left(p_{1}\right) \mathrm{d} p_{1}
$$

The second derivative w.r.t. $\tilde{d}$ and the mixed $\tilde{\gamma}, \tilde{d}$ second derivative are given by similar expressions. The second derivative of $\tau$ w.r.t. $\tilde{\gamma}$ is given by

$$
\frac{\delta^{2} \tau}{\delta \tilde{\gamma}^{2}} \delta \tilde{\gamma} \delta \tilde{\gamma}^{\prime}=\int_{0}^{\tilde{d}} \frac{3}{2} p_{1}^{4}\left(1-\tilde{\gamma}(s) p_{1}^{2}\right)^{-\frac{5}{2}} \delta \tilde{\gamma}(s) \delta \tilde{\gamma}^{\prime}(s) \mathrm{d} s .
$$

Now suppose $\delta \tilde{\gamma}^{\prime}=\delta \tilde{\gamma} \neq 0$, and

$$
\int_{0}^{\tilde{d}} \delta \tilde{\gamma}(s) \mathrm{d} s=0
$$

Then the second term on the right hand side of (21) vanishes and $\frac{\delta^{2} \hat{K}}{\delta \tilde{\gamma}^{2}} \delta \tilde{\gamma} \delta \tilde{\gamma}$ is given by a positive constant times

$$
\int_{-\alpha}^{\alpha} p_{1}^{4}\left(1-\tilde{\gamma} p_{1}^{2}\right)^{-\frac{5}{2}}\left(\tau\left(p_{1}\right)-\tau^{*}\left(p_{1}\right)\right) \mathrm{d} p_{1} .
$$

Since $\frac{\delta K}{\delta(\tilde{d}, \tilde{\gamma})}=0$, we have

$$
\begin{array}{r}
\int_{-\alpha}^{\alpha}\left(1-\tilde{\gamma} p_{1}^{2}\right)^{-\frac{1}{2}}\left(\tau\left(p_{1}\right)-\tau^{*}\left(p_{1}\right)\right) \mathrm{d} p_{1}=0 \\
\int_{-\alpha}^{\alpha} p_{1}^{2}\left(1-\tilde{\gamma} p_{1}^{2}\right)^{-\frac{3}{2}}\left(\tau\left(p_{1}\right)-\tau^{*}\left(p_{1}\right)\right) \mathrm{d} p_{1}=0 .
\end{array}
$$

This implies that $\tau-\tau^{*}$ has at least two zeroes for $p_{1}>0$. We show below that $\tau-\tau^{*}$ in fact has at most two zeroes, and that in that case it has signs,,-+- in this order from $p_{1}=0$ to $p_{1}=\alpha$. It then follows that (23) is negative and therefore the Hessian has a negative eigenvalue.

Remains to show that $\tau-\tau^{*}$ has at most two zeroes and signs,,-+- from $p_{1}=0$ to $p_{1}=\alpha$. In fact we show that when $q>0$ is such that $\tau^{*}(q)=\tau(q)$ and $\frac{\partial \tau^{*}}{\partial p_{1}}(q) \geq \frac{\partial \tau}{\partial p_{1}}(q)$, then the higher order derivatives of $\tau^{*}, \tau$ satisfy $\frac{\partial^{k} \tau^{*}}{\partial p_{1}^{k}}(q) \geq \frac{\partial^{k} \tau}{\partial p_{1}^{k}}(q)$. Since $\tau^{*}$ and $\tau$ are analytic and $\tau^{*} \neq \tau$ this implies that $\tau^{*}\left(p_{1}\right)>\tau\left(p_{1}\right)$ for $p_{1}>q$.

The traveltimes $\tau(q), \tau^{*}(q)$ are given by

$$
\begin{aligned}
\tau(q) & =2 \tilde{d}\left(1-\tilde{\gamma} q^{2}\right)^{-\frac{1}{2}} \\
\tau^{*}(q) & =\int_{0}^{\tilde{d}^{*}} 2\left(1-\tilde{\gamma}^{*}(s) q^{2}\right)^{-\frac{1}{2}} \mathrm{~d} s .
\end{aligned}
$$


Rewriting $\tau^{*}$ using the measure $\left(\tilde{\gamma}^{*}\right)_{*} I_{\left[0, \tilde{d}^{*}\right]}$ defined in (8), the equation $\tau^{*}(q)=\tau(q)$ reads

$$
\int_{y_{\min }}^{y_{\max }}\left(1-y q^{2}\right)^{-\frac{1}{2}}\left(\left(\tilde{\gamma}^{*}\right)_{*} I_{\left[0, \tilde{d}^{*}\right]}\right)(y) \mathrm{d} y \geq \tilde{d}\left(1-\tilde{\gamma} q^{2}\right)^{-\frac{1}{2}} .
$$

Similarly the equation $\frac{\partial \tau^{*}}{\partial p_{1}}(q) \geq \frac{\partial \tau}{\partial p_{1}}(q)$ is equivalent to the following equation, with $k=1$,

$$
\int_{y_{\min }}^{y_{\max }}\left(1-y q^{2}\right)^{-\frac{1}{2}-k}\left(\left(\tilde{\gamma}^{*}\right)_{*} I_{\left[0, \tilde{d^{*}}\right]}\right)(y) \mathrm{d} y=\tilde{d}\left(1-\tilde{\gamma} q^{2}\right)^{-\frac{1}{2}-k} .
$$

By examining the higher derivatives of $\tau^{*}, \tau$ we see that it is sufficient to show that (25) is also true for $k \geq 2$. Define a diffeomorphism $y \mapsto z=\left(1-y q^{2}\right)^{-1}$, then there is some positive measure $v(z)$ supported in $z>0$ such that the left hand side of (25) equals

$$
\int_{z_{\min }}^{z_{\max }} z^{k} v(z) \mathrm{d} z
$$

Let $z_{0}=\left(1-\tilde{\gamma} q^{2}\right)^{-1}$ then $(25)$ reads for $k=1$ (using (24))

$$
\int_{z_{\min }}^{z_{\max }} z v(z) \mathrm{d} z \geq z_{0} \int_{z_{\min }}^{z_{\max }} v(z) \mathrm{d} z .
$$

Since $v$ is positive and supported in $z>0$ this implies that

$$
\int_{z_{\min }}^{z_{\max }} v(z) z^{k} \mathrm{~d} z \geq z_{0}^{k} \int_{z_{\min }}^{z_{\max }} v(z) \mathrm{d} z
$$

for $k \geq 2$ and hence that (25) is true for $k \geq 2$. This concludes the $p_{1}$ case.

For the same result using the $x_{1}$ coordinate the functional derivative of (15) needs to be taken. Because $\delta \tilde{\gamma}$ is chosen satisfying (22) the term involving $\frac{\delta X_{1}}{\delta \tilde{\gamma}} \delta \tilde{\gamma}$ vanishes. It follows that mutatis mutandis the proof applies in this case as well.

Let us now consider the differential semblance objective functional. We will use the modified depth coordinate s from (3) and the corresponding depth and medium $\tilde{d}, \tilde{\gamma}$. In the differential semblance functional the derivative of traveltime at depth $\tilde{d}^{*}$ is compared with the derivative of traveltime at depth $\Gamma\left(x_{1}, \tilde{d}^{*}\right)$ that depends on both the real and the guessed medium and is defined by

$$
T\left(x_{1} ; \Gamma\left(x_{1}, \tilde{d}^{*}\right), \tilde{\gamma}\right)=T\left(x_{1} ; \tilde{d}^{*}, \tilde{\gamma}^{*}\right) .
$$

We will use the shorthand notation $T\left(x_{1}, s\right)=T\left(x_{1} ; s, \tilde{\gamma}\right), T^{*}\left(x_{1}, s\right)=T\left(x_{1} ; s, \tilde{\gamma}^{*}\right)$. The differential semblance functional depends only on the medium coefficient $\tilde{\gamma}$ (or $\gamma$ ). We will not discuss here how it is evaluated from data, we just use that for a single reflector it is given by (see [5])

$$
J(\tilde{\gamma})=\int w^{*}\left(x_{1}, \tilde{d}^{*}\right)\left(\frac{\partial T}{\partial x_{1}}\left(x_{1}, \Gamma\left(x_{1}, \tilde{d}^{*}\right)\right)-\frac{\partial T^{*}}{\partial x_{1}}\left(x_{1}, \tilde{d}^{*}\right)\right)^{2} \mathrm{~d} x_{1},
$$


where $w^{*}$ is positive function depending only the real medium. After $\tilde{\gamma}$ is reconstructed, the depth of the reflector can be found by imaging the data. A similar definition can be made with $x_{1}$ replaced by $p_{1}$, the corresponding $p_{1}$ domain differential semblance functional will be denoted by $\hat{J}(\tilde{\gamma})$.

In the next theorem it is assumed that $\tilde{\gamma}$ is nonconstant for $s$ less than

$$
\Gamma_{\min }=\min _{x_{1} \in[-\hat{\alpha}, \hat{\alpha}]} \Gamma\left(x_{1}, \tilde{d}^{*}\right) .
$$

When $J$ has a stationary point then $x_{1} \mapsto\left(\frac{\partial T}{\partial x_{1}}\left(x_{1}, \Gamma\left(x_{1}, \tilde{d}^{*}\right)\right)-\frac{\partial T^{*}}{\partial x_{1}}\left(x_{1}, \tilde{d}^{*}\right)\right)$ must change sign somewhere, and this gives bounds (depending on the bounds of $c$ ) on $\Gamma\left(x_{1}, \tilde{d}\right)-\tilde{d}$, so this condition is not too restrictive.

Theorem 4.3 Assume that $\tilde{\gamma}$ is nonconstant for $s<\Gamma_{\min }$. Then

$$
\frac{\delta J}{\delta \tilde{\gamma}}(\tilde{\gamma})=0
$$

implies that

$$
J(\tilde{\gamma})=0
$$

The corresponding statement for the $p_{1}$ domain functional $\hat{J}$ is also true.

Proof The derivative of $J$ with respect to $\tilde{\gamma}$ is given by

$$
\frac{\delta J}{\delta \tilde{\gamma}}=2 \int w^{*}\left(x_{1}, \tilde{d}\right)\left(\frac{\partial T}{\partial x_{1}}\left(x_{1}, \Gamma\left(x_{1}, \tilde{d}\right)\right)-\frac{\partial T^{*}}{\partial x_{1}}\left(x_{1}, \tilde{d}\right)\right) \frac{\delta}{\delta \tilde{\gamma}}\left(\frac{\partial T}{\partial x_{1}}\left(x_{1}, \Gamma\left(x_{1}, \tilde{d}\right)\right)\right) \mathrm{d} x .
$$

Therefore it is sufficient to show that the set

$$
\left\{\delta \tilde{\gamma} \frac{\delta}{\delta \tilde{\gamma}}\left(\frac{\partial T}{\partial x_{1}}\left(x_{1}, \Gamma\left(x_{1}, \tilde{d}\right)\right)\right) \mid \delta \tilde{\gamma} \in C_{0}^{\infty}\left(\left[0, \Gamma_{\min }\right]\right)\right\}
$$

is dense in $C_{\text {odd }}^{\infty}([-\hat{\alpha}, \hat{\alpha}])$. For $p_{1}$-domain this is true for

$$
\left\{\delta \tilde{\gamma} \frac{\delta}{\delta \tilde{\gamma}}\left(\frac{\partial \tau}{\partial p_{1}}\left(p_{1}, \hat{\Gamma}\left(p_{1}, \tilde{d}\right)\right)\right) \mid \delta \tilde{\gamma} \in C_{0}^{\infty}\left(\left[0, \hat{\Gamma}_{\min }\right]\right)\right\}
$$

where $\hat{\Gamma}$ is the $p_{1}$ equivalent of $\Gamma$.

We first show the result for $\hat{J}$ ( $p_{1}$ domain). From the equivalent of (26) we find

$$
\frac{\delta \Gamma}{\delta \tilde{\gamma}} \delta \tilde{\gamma}=-\left(\frac{\partial T}{\partial s}\left(p_{1}, \Gamma\left(p_{1}, \tilde{d}\right)\right)\right)^{-1} \frac{\delta T}{\delta \tilde{\gamma}} \delta \tilde{\gamma}\left(p_{1}, \Gamma\left(p_{1}, \tilde{d}\right)\right)
$$

We find

$$
\begin{aligned}
\delta \tilde{\gamma} \frac{\delta}{\delta \tilde{\gamma}}\left(\frac{\partial T}{\partial p_{1}}\left(p_{1}, \Gamma\left(p_{1}, \tilde{d}\right)\right)\right)= & \frac{\partial}{\partial p_{1}} \frac{\delta T}{\delta \tilde{\gamma}} \delta \tilde{\gamma}\left(p_{1}, \Gamma\left(p_{1}, \tilde{d}\right)\right) \\
& -\frac{\partial^{2} T}{\partial p_{1} \partial s}\left(\frac{\partial T}{\partial s}\left(p_{1}, \Gamma\left(p_{1}, \tilde{d}\right)\right)\right)^{-1} \frac{\delta T}{\delta \tilde{\gamma}} \delta \tilde{\gamma}\left(p_{1}, \Gamma\left(p_{1}, \tilde{d}\right)\right)
\end{aligned}
$$


Define $h\left(p_{1}\right)=\frac{\partial^{2} T}{\partial p_{1} \partial s}\left(\frac{\partial T}{\partial s}\left(p_{1}, \Gamma\left(p_{1}, \tilde{d}\right)\right)\right)^{-1}$, then this is equal to

$$
e^{\int_{0}^{p_{1}} h\left(p_{1}^{\prime}\right) \mathrm{d} p_{1}^{\prime}} \frac{\partial}{\partial p_{1}}\left(e^{-\int_{0}^{p_{1}} h\left(p_{1}^{\prime}\right) \mathrm{d} p_{1}^{\prime}} \frac{\delta T}{\delta \tilde{\gamma}}\right) .
$$

It follows from similar arguments as in the proof of Theorem 3.2 that

$$
\left\{\frac{\delta T}{\delta \tilde{\gamma}} \delta \tilde{\gamma} \mid \delta \tilde{\gamma} \in C_{0}^{\infty}\left(\left[0, \Gamma_{\min }\right]\right)\right\}
$$

is dense in

$$
\left\{f \in C_{\text {even }}^{\infty}([-\alpha, \alpha]) \mid f(0)=0\right\}
$$

by applying Lemma 3.4 to (17), and then applying Lemma 3.5 etc. The operator $\frac{\partial}{\partial p_{1}}$ is a homeomorphism from the set (30) to $C_{\text {odd }}^{\infty}([-\alpha, \alpha])$. From (29) (since $p_{1} \mapsto \int_{0}^{p_{1}} h\left(p_{1}^{\prime}\right) \mathrm{d} p_{1}^{\prime}$ is smooth, even and greater then zero), it follows that $(28)$ is dense in $C_{\text {odd }}^{\infty}([-\alpha, \alpha])$. This finishes the proof of the $p_{1}$ case.

The $x_{1}$ case can be proved by modifying the proof for the $p_{1}$ case in a similar way as in the proof of Theorem 3.2, using (15) and the fact that the map $x_{1} \mapsto p_{1}=$ $\frac{\partial T}{\partial x_{1}}\left(x_{1}, \Gamma\left(x_{1}, \tilde{d}\right)\right)$ is a diffeomorphism.

\section{Acknowledgments}

The author would like to thank W.W. Symes for many useful discussions. This work is supported in part by The Rice Inversion Project.

\section{References}

[1] G. Beylkin. The inversion problem and applications of the generalized Radon transform. Comm. Pure Appl. Math., 37:579-599, 1984.

[2] William W. Symes. A differential semblance algorithm for the inverse problem of reflection seismology. Comput. Math. Appl., 22(4-5):147-178, 1991.

[3] K. M. Al-Yahya. Velocity analysis by iterative profile migration. Geophysics, 54:718-729, 1989.

[4] Hervé Chauris. Analyse de vitesse par migration pour l'imagerie des structures complexes en sismique réflexion. PhD thesis, École des Mines de Paris, 2000.

[5] William W. Symes. All stationary points of differential semblance are asymptotic global minimizers: layered acoustics. Technical Report TR99-08, CAAM Dept., Rice University, Houston, 1999. http://www.caam.rice.edu/.

[6] T. N. Bishop, K. P. Bube, R. T. Cutler, R. T. Langan, P. L. Love, J. R. Resnick, R. T. Shuey, D. A. Spindler, and H. W. Wyld. Tomographic determination of velocity and depth in laterally varying media. Geophysics, 50:903-923, 1985.

[7] Sven Ivansson. Some remarks concerning seismic reflection tomography and velocity analysis. Geophys. J. R. Astr. Soc., 87:539-557, 1986.

[8] C. Hewitt Dix. Seismic velocities from surface measurements. Geophysics, 20:68-86, 1955.

[9] M. L. Gerver and V. M. Markushevich. On the characteristic properties of travel-time curves. Geophys. J. R. Astr. Soc., 13:241-246, 1967. 
[10] Sven Ivansson. Some considerations of uniqueness and nonuniqueness in seismic reflection tomography. In Technical Program, 56th Annual SEG Meeting, pages 542-545, Tulsa, OK, 1986. Society of Exploration Geophysicists.

[11] K. P. Bube and J. R. Resnick. Well-determined and poorly determined features in seismic reflection tomography. In Technical Program, 54th Annual SEG Meeting, pages 717-719, Tulsa, OK, 1984. Society of Exploration Geophysicists.

[12] K. P. Bube, D. B. Jovanovich, R. T. Langan, J. R. Resnick, R. T. Shuey, and D. A. Spindler. Well-determined and poorly determined features in seismic reflection tomography: Part II. In Technical Program, 55th Annual SEG Meeting, pages 608-610, Tulsa, OK, 1985. Society of Exploration Geophysicists.

[13] Christof Stork and Robert W. Clayton. Analysis of the resolution between ambiguous velocity and reflector position for traveltime tomography. In Technical Program, 56th Annual SEG Meeting, pages 545-550, Tulsa, OK, 1986. Society of Exploration Geophysicists.

[14] Kenneth P. Bube, Robert T. Langan, and Jeffrey R. Resnick. Unique determination of reflector depths in seismic reflection tomography. In Technical Program, 59th Annual SEG Meeting, pages 918-921, Tulsa, OK, 1989. Society of Exploration Geophysicists.

[15] Christof Stork. Singular value decomposition of the velocity-reflector depth tradeoff, part 2: Highresolution analysis of a generic model. Geophysics, 57:933-943, 1992.

[16] Kenneth P. Bube. Uniqueness of reflector depths and characterization of the slowness null space in linearized seismic reflection tomography. SIAM J. Appl. Math., 55(1):255-266, 1995.

[17] Keiiti Aki and Paul G. Richards. Quantitative Seismology: Theory and Methods, volume 2. Freeman, San Francisco, 1980. 